MATEC Web of Conferences 31, 12002 (2015)

DOI: $10.1051 /$ matecconf/ 20153112002

(C) Owned by the authors, published by EDP Sciences, 2015

\title{
Research on 3D reconstruction of concrete
}

\author{
Shi Pan-fei, Gao yang, Yang yue-fei, Liu Jing-hong \\ Agricultural University of He bei, China
}

\begin{abstract}
In order to associate with using acoustic emission, ultrasonic or other means to locate damage of concrete, based on the concrete CT image information, the research of 3D reconstruction of concrete crack structure was completed by using Amira 5.2.13D reconstruction software. Experiments showed that: three dimensional reconstruction of concrete can reflect the real crack structure, and distribution of aggregate concrete of three dimensional model established was basically in accordance with the original CT image, which laid the good foundation to simulate and analysis by using ANSYS finite element software in the future.
\end{abstract}

\section{Introduction}

Concrete is a complex multiphase composite materials, $\mathrm{w}$ hich mainly consists of mortar, stone, porous compositio n. CT is short for Computerized Tomography, and CT ex periments have great advantage in non-destructive concr ete detection, and real-time observation of concrete crack $\mathrm{s}$ in materials changes [1]. By scanning cross-section of co ncrete specimen with industrial CT, concrete $\mathrm{CT}$ image ca $\mathrm{n}$ be obtained, from which we can establish a real 3D mod el. A great many scholars applied CT technology to the re search and analysis of concrete materials. Su Sheng[2] go $\mathrm{t}$ a more real concrete aggregate model by conducting thre e dimensional reconstructions respectively based on surfa ce data and volume data, which made great contribution $\mathrm{t}$ $o$ the study of the characteristics of the internal aggregate $t$ hat influence on the performance of concrete. Jiang Yuan [3] proposed that directly using the original $\mathrm{CT}$ image in DICOM format rather than the image format conversion $t$ o complete the construction of concrete structure, it made the internal structure of concrete more real. Hao shu-liang [4] completed the three-dimensional reconstruction of the concrete by MATLAB software, basing on the CT image of concrete. Tian Wei [5] used three-dimensional image pr ocessing software mimics to realize the $3 \mathrm{D}$ reconstruction of the concrete structure, at the same time used ANSYS s oftware to simulate and analyze, and achieved good result s. Liu Han-kun [6] did 3D reconstruction of CT images b y using MIMICS software, a three-dimensional geometric model of concrete, and used Abaqus finite element softw are to complete the numerical simulation analysis, finally achieved good results. Amira[7] is a modelling software s ystem, and furthermore, it can realize 3D visualization. By using the Amira to conduct the three dimensional reconst

Corresponding author: Shi Pan fei; e-mail:991070119@qq.com ruction of cylindrical concrete crack, the 3D visualization model of concrete crack was been done.

\section{CT image acquisition}

\subsection{The test specimen preparation}

In the test of concrete specimens with information such as shown in table 1.

Table 1.The mix of concrete' in the test

material Amount of per cubic concrete

\begin{tabular}{rrrr}
\hline Cement/kg & Water/kg & Sand $/ \mathrm{kg}$ & Gravel $/ \mathrm{kg}$ \\
327 & 189 & 755 & 1133 \\
\hline
\end{tabular}

\subsection{Scanning device}

CT detection system in the test is ACTIS300-320/225X fr om the State Key laboratory in China University of Minin $\mathrm{g} \&$ Technology (Beijing) [8], and the image size in pixel $\mathrm{s}$ is $500 * 500$. The scanning thickness is $0.2 \mathrm{~mm}$. Moreover, the specimen specification is a cylinder with a radius of 5 $0 \mathrm{~mm}$ and a high of $190 \mathrm{~mm}$. DS2 series full information o $f$ acoustic emission signal analyzed to complete the AE da ta acquisition of the whole loading process. The relevant a coustic emission parameter setting, threshold values of 10 0dB, PDT: 150us, HDT: 300us, HLT: 500us. The related $\mathrm{t}$ echnical indicators: 8 channel $3 \mathrm{MHZ}$, sampling rate, data 
collection methods: 4 channel synchronous data acquisitio n, RS-35C integrated front sensor: amplifier gain: 100 tim es.

The specimens were carried out in 6 stages of scannin $\mathrm{g}$, including the initial phase, as shown in Table 2.

Table 2. The load corresponding to the 6 scanning time of the specimen

\begin{tabular}{cccccc}
\hline $\begin{array}{c}\text { The } \\
\text { first }\end{array}$ & $\begin{array}{c}\text { The } \\
\text { second }\end{array}$ & $\begin{array}{c}\text { The } \\
\text { third }\end{array}$ & $\begin{array}{c}\text { The } \\
\text { Fourth }\end{array}$ & $\begin{array}{c}\text { The } \\
\text { fifth }\end{array}$ & $\begin{array}{c}\text { The } \\
\text { sixth } \\
\text { scan }\end{array}$ \\
scan & scan & scan & scan & scan \\
\hline Not & $22.3 \%$ & $44.8 \%$ & $67.2 \%$ & $89.6 \%$ & peak \\
loaded & peak & peak & peak & peak & intensit \\
intensit & intensit & intensit & intensit & $\begin{array}{c}\mathrm{y} \\
(\mathrm{kn})\end{array}$ \\
0 & $\mathrm{y}(\mathrm{kn})$ & $\mathrm{y}(\mathrm{kn})$ & $\mathrm{y}(\mathrm{kn})$ & $\mathrm{y}(\mathrm{kn})$ & 134 \\
\hline
\end{tabular}

Schematic diagram of experimental system was as shown in Figure 1.

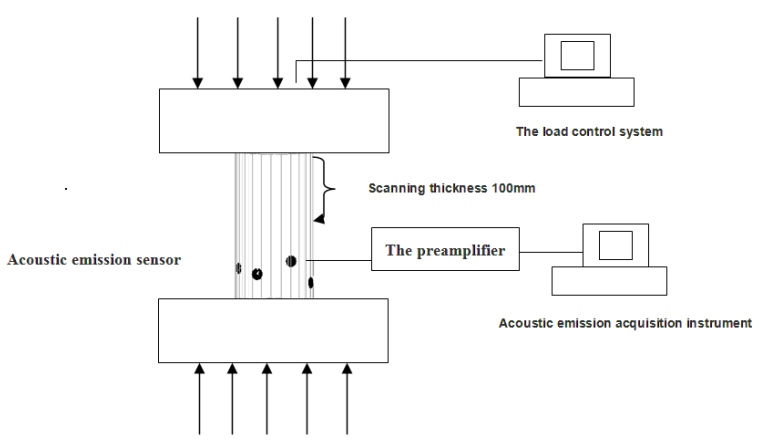

Figure 1. schematic diagram of experimental system

\subsection{The result of the experiment}

The specimen loading pressure- time curve was as shown in Figure 2.

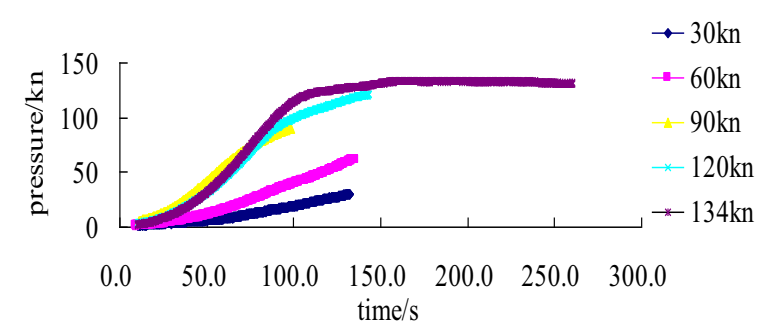

Figure 2 pressure displacement diagram

998 CT images were obtained. Select one section under different stress phase as shown in Figure 3.

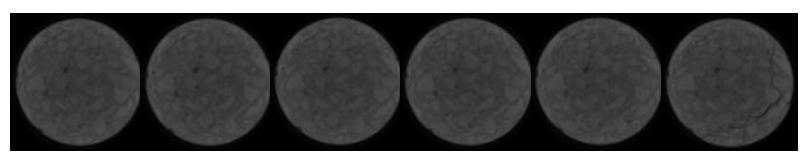

Fig.3 CT scanning image of the failure process of specimen und er uniaxial compression

There is no obvious changes and crackle in the first stages until the peak pressure.

The Amira software extracted the crack porosity as shown in Figure 4.

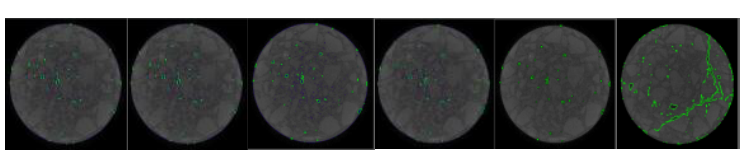

Figure 4: Pore threshold segmentation map

Concrete pore and crack is a green in figure 3, and pore crack changed more clearly than $\mathrm{CT}$ original images through the Amira software extracted pore crack.

\section{Three dimensional reconstruction of concrete pore based on CT technology}

By using CTA's image data set, Arima provides several means to get three-dimensional shape impressions, such as orthogonal slices, the subsurface to display the body surface higher than the threshold selected by user, volume rendering without data segmentation.

Both the generation of surface model and volume measurement are required for image segmentation. The so-called image segmentation is to assign each pixel a label to indicate the area of the pixel existence and its material properties [9]. Arima provides a rich set of tools that can be used for manual image segmentation, such as Brush, Lasso, Magic Wand and Blow Tool.

By connecting data with OrthoSlice model, the section image of concrete can be seen, and by adjusting Window Data, this paper can determine the segmentation threshold of each material suitable. Through several adjustments, this paper found that the number of each material CT in concrete sections from the experiment isn't so obvious, and if we just utilize automatic threshold segmentation, it's difficult to distinguish the mortar in edge and stone in the middle, so are the mortar in the middle and part of the crack. Considering the manual segmentation spends lots of manual labour and time, thus, this paper chose the means of manual intervention combined with threshold segmentation. Import data file, connect Image Segmentation Editor, and then add mortar, crack, stone, and pore, finally, give them individual colours. Choose the center region of the image, then click the plus symbol of "Crack"and"Selection" in the material list in turn. Thus, 
the center region in the pictures is assigned to Crack, and the Crack is locked. Distribute the mortar in the edge by adjusting the range of the threshold. So we can avoid mistaking the stone in the middle for mortar as the central region was locked by Crack. Distribute the pixel to all stones in the same way. With the same methods of operation, separate part of the mortar in the middle from crash part to make the main crack more clearly. Then filter and smooth all the image slices, image segmentation as shown in Figure 5.

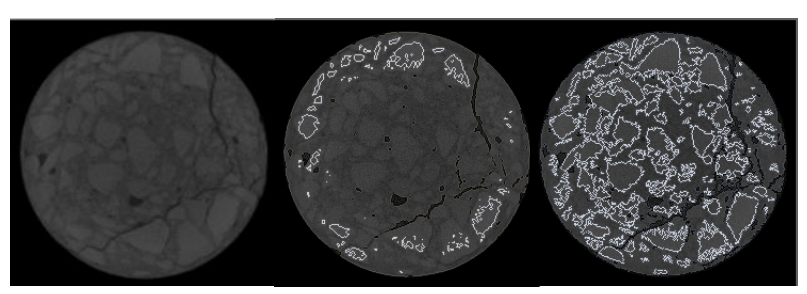

Figure 5: From left to right: the original CT image, automatic threshold segmentation, manual and automatic segmentation

Stones were surrounded by white, mortar by gray and major crack by black. The middle image was been done by threshold segmentation, and the central stone was divided falsely into mortar, because of the gray value of mortar in the edge similar to the central stone's. The right graph done by the combination of human and Computer is similar to the original CT image and more reasonable.

Connecting the Resample module for data re-samplin $\mathrm{g}$, the resolution will reduce some, but it can reduce the co mputation time and improve the rendering effect. Connect with the SurfaceGen module to complete the surface of $t$ he gap model; finally this paper can observe three dimensi onal reconstruction models through Surface View module. It can be arbitrarily combined display, such as the spatial relations between mortar and stones, the spatial relations

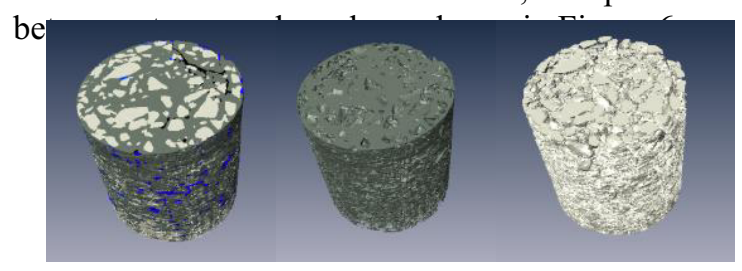

Figure 6: The first row from left to right: Overall, mortar, aggregate

Distribution of aggregate concrete of three dimensiona 1 model established was basically in accordance with the o riginal CT image. Through software in statistical, the volu me of each component is roughly the same with the mix $r$ atio of the text block, and the special form of crack is clea r. By transparent process with software, it can realize th e visualization of crack, which truly displays the internal c rack of concrete material. By the reconstruction process, $i$ $\mathrm{t}$ can be seen that the surrounding mortar is slightly larger than the central mortar in density. From the three dimensi onal reconstruction, it can be seen that the aggregate distri butes quite evenly. The crack connects part of the initial $p$ ore, and it mainly appears in the edge, not inverted triangl e. The cylindrical test block was eccentric compression, w hich can be caused by the uneven surface.

\section{The acoustic emission damage localiza tion}

Acoustic emission signal analyzer used three-dimensiona 1 positioning method for concrete specimen's damage loca tion, according to the time difference of the acoustic emiss ion channel signal, acoustic emission wave, and the positi on of the probe to determine the coordinate position of da mage point. To extract channel time difference of acoustic emission signal is important, and the related parameter is the threshold value and the setup of the time parameter. Th e threshold is setup up between the maximum amplitude a nd the amplitude of noise. Time parameters include pea $\mathrm{k}$ definition of time (PDT), impact time and impact definit ion (HDT) blocking time (HLT). The definition of time is used to verify the impact signals the end, and if set too sho $\mathrm{rt}$, a signal will be divided into several signal, and if too lo ng, several statistical signal will be took as a signal. Impac t of the locking time is used to avoid the statistics to the re flected wave signal of damage. In practice, for the damag e location of materials, especially like concrete composite materials, not only the threshold value and the time param eters were taken into account settings, it should consider $t$ he two parameters. The acoustic emission damage localiza tion accuracy imitated by the concrete is an objective fac t. Considering ratio and the number of the damage locatio $\mathrm{n}$ in the crack area, computing speed, when the threshol $\mathrm{d}$ values is $100 \mathrm{~dB}$, PDT: $150 \mathrm{us}$, HDT: $300 \mathrm{us}$, HLT: $500 \mathrm{u}$ $\mathrm{s}$, crack damage orientation clear and relatively ideal. Th e acoustic emission damage location diagram was as show $\mathrm{n}$ in figure 7.
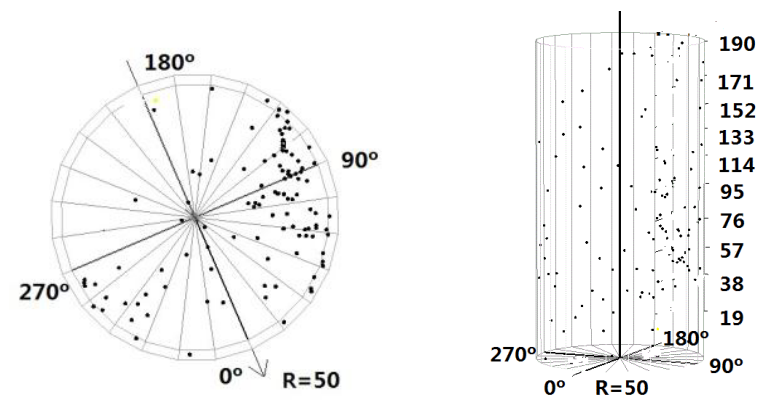

Figure 7: The AE damage location map

The acoustic emission damage location damage failure surface position was clear, with which $3 \mathrm{D}$ reconstructio $\mathrm{n}$ model of concrete crack shows good consistency, and ac 
curately track the damage location, meet the engineering $r$ equirements for finding the "weak" in the material or stru cture.

\section{Conclusions}

Based on the above analysis, we can draw the following conclusions:

This paper can get the CT slice image ideally by scann ing the cylindrical concrete and through Amira-3D visuali zation software on personal computers, and realized the th ree-dimensional reconstruction of crack in concrete struct ure. compared With previous methods to do $3 \mathrm{D}$ reconstruc tion of concrete based on CT image, this paper improved $i$ $\mathrm{n}$ this place: when the picture quality is not too good, this paper completed the image segmentation by human-comp uter interaction, which was better than the Matlab model directly based on the threshold segmentation for 3D recon struction; this model was more intuitive, which realized th e combination display of various materials and transparent display;Utilize various methods to realize three-dimensio nal impression. The practice showed that, we only need le ss time to complete the three-dimensional reconstruction with Amira software. Although the three-dimensional crac $\mathrm{k}$ model reconstruction is not ideal, and the three-dimensi onal reconstruction can reflect crack structure of concrete more real. Moreover, damage localization of concrete can be associated with acoustic emission or ultrasonic and oth er means of mutual aided verification. The established thr ee dimensional model of distribution of aggregate concret $\mathrm{e}$ is basically in accordance with the original CT image; it also laid a good foundation for the introduction of ANSYS finite element software to simulation calculation. Based o $\mathrm{n}$ CT image information, crack model reconstruction by $\mathrm{u}$ sing the 3D Amira reconstruction software can reflect th e real structure of concrete crack, which was been used $f$ or acoustic emission location of damage on the concrete $\mathrm{m}$ utual aided verification.

But by the limit of software, so image segmentation ha $\mathrm{d}$ not been a unified standard, and this paper also has the $\mathrm{f}$ ollowing shortcomings to be improved:

A) Automatic threshold segmentation has high requirem ents on the picture, but the manual segmentation is $\mathrm{m}$ ore subjectivity. the segmentation effect by using the method of combination of human and computer is $i$ mproved, and the overall regularity is no problem, bu $t$ the data accuracy will be affected, which is not cond ucive to the next quantitative analysis

B) amount of initial crack is differ from the statistical val ues, there may being reasons: measurement error; th e pore distribution may also have an impact, because the pore volume of statistics is owned to the local blo ck; the main reason is that Amira statistical software $r$ equirement for the volume of pore sealing, so the surr ounding open pores or not closed pores in the test blo ck will not be statistics, resulting that pore statistics is not comprehensive enough; Some micro pore was n ot extracted in the process.

\section{Acknowledgments}

Thanks the Key Fund Project in Education Department of Hebei Province（ZD2014073） and the Technology Fund Projects of Hebei Agricultural University（LG20140304) for their supporting. Tremendous thanks owned to several graduate classmates for their assistant during the test and analysis of the work summarized here.

\section{References}

1. H.Q. Chen, W.H. Ding, Y.B. Pu. Real time observa tion on meso fracture process of concrete using X-ray $\mathrm{CT}$ under uniaxial compressive condition $[\mathrm{J}]$. Journal of hydraulic engineering, 2006, 37 (9): 1044-1050.

2. S. Su, D.J. Shen, W.B. Lv. Research and Application on 3-D Reconstructed Technology of Random Distrib ution of Aggregates in Fully-graded Concrete [J]. Jian gxi science, 2007, 25 (5): 522-525531.

3. Y. Jiang, W. Bai, Y.L. Qi. Reconstruction of 3D Mo del of Concrete Mesa structure with CT Original Dat a [J]. Journal of China Three Gorges University: Natu ral Science Edition, 2008,30 (1): 52-55.

4. S.L. Hao, F.N. Dang, H.Q. Chen. Three dimension re construction of concrete meso-structure based on CT images by ANSYS[J]. The concrete, 2009 (3): 1315.

5. W. Tian, F.N. Dang, H.Q. Chen. Research on three-d imension reconstruction technology of concrete based on CT images [J]. Journal of Sichuan University (En gineering Science Edition), 2010, 42 (6): 12-16.)

6. H.K. Liu, J. Li. Non localization meso damage mod el of concrete under Uniaxial tension state [J]. Journal of Tong ji University, 2014, (02): 203-209.

7. Amira 4.1Manual(PDF).2005.MERCURY (amira use $r$ guide).

8. Liu J.H. Liu, Y.D. Jiang. Breakage process analysis o f rock on uniaxial compression CT tests [J]. Journal o f Agricultural University of Hebei, 2008, 31 (4): 112115.

9. Levoy M.Efficient Ray Tracing of volume Data.AC M Transaction on Graphics 1990,9:245-261 